\title{
Interviews ausstellen
}

\section{Reflexionen zum performativen Umgang mit qualitativen Daten in wissenschaftlichen Ausstellungen ${ }^{1}$}

\author{
Günter Mey
}

Journal für Psychologie, 28(1), 108-133

https://doi.org/10.30820/0942-2285-2020-1-108

CC BY-NC-ND 3.0 DE

www.journal-fuer-psychologie.de

\section{Zusammenfassung}

In dem Beitrag werden Möglichkeiten erörtert, um Ergebnisse aus qualitativen Forschungsstudien in Ausstellungen zu präsentieren. Nach einführenden Anmerkungen zur performativen Sozialwissenschaft, die für eine (nicht fach-)öffentliche Dissemination künstlerischästhetische Mittel nutzt, folgen einige Beispiele von Ausstellungen. Der Fokus liegt dabei auf der Verwendung von Interviewdaten und ihrer Transformation als aufbereitete Dossiers, Zitatenkollektionen, Videocollagen und anderen Präsentationsmodi. Im Anschluss werden forschungsethische Überlegungen ebenso wie die Frage der Geltungsbegründung diskutiert.

Schlüsselwörter: Performative Sozialwissenschaft, qualitative Forschung, Wissenschaftsausstellungen, Interviews, Gütekriterien

\section{Summary}

Exhibit Interviews. Reflections on presenting qualitative data in scientific exhibitions

The paper discusses ways to present results from qualitative research studies in exhibitions to disseminate science for the (non-professional) public. After introductory comments on performative social science some examples of exhibitions follow. The focus is on the use of interview data and its transformation as quotation collections, video collages and other presentation modes. Subsequently, research ethical considerations as well as the question of quality criteria are discussed.

Keywords: performative social science, qualitative research, science exhibitions, interviews, quality criteria 


\section{$1 \quad$ Einleitung}

Ausstellungen, eines der ältesten Formate, um dem Auftrag der Wissensvermittlung und Bildung zu folgen, stellen traditionell eine besondere Möglichkeit dar, Forschungsergebnisse für eine interessierte Öffentlichkeit zu präsentieren. Heutige Wissenschaftsausstellungen, wie sie etwa im Deutschen Hygiene Museum in Dresden oder im Museum für Kommunikation in Berlin, ebenso in vielen städtisches Einrichtungen und diversen lokalen Foren, gezeigt werden, stehen dabei für sich wandelnde Präsentationskonzepte, auch und gerade zu human-, sozial- und kulturwissenschaftlichen Fragestellungen. Es geht nicht mehr länger um das »Public Understanding of Science«, bei dem Wissenschaft in Form von Fakten und Produkten präsentiert wird, vielmehr wird - im Sinne eines »Public Understanding of (Current) Research « - ein Verständnis für eine sich im Prozess befindliche Forschung geschaffen (Field und Powell 2001). Damit verbindet sich auch der Anspruch, Ausstellungen so zu kuratieren, dass sie zu Orten werden, an denen gelernt, entdeckt und konstruiert werden kann - indem sie den Besuchenden eröffnen, ihre eigenen Erfahrungen mithilfe der Themen und Objekte der Ausstellung zu erweitern und zu hinterfragen (Lepenies 2003).

Interessanterweise finden sich wenig explizite Hinweise auf Ausstellungen im Rahmen der performativen Sozialwissenschaft, bei der eine neue Form der Dissemination wissenschaftlicher Erkenntnisse - zumeist aus qualitativen Forschungsstudien - unter Rückgriff auf künstlerische Ausdruckformen entsteht. Abhandlungen zu Ausstellungen (z. B. Church 2008; Sullivan 2010, 207-10) nehmen in der mittlerweile vielfältig verfügbaren Literatur nur einen Randplatz ein - verglichen mit den vielen Beiträgen zu den Präsentationsmodi, bei denen Forschungsergebnisse als Autoethnografie, Fiction oder Poetik angelegt oder in Musik-, Tanz- und Theateraufführungen umgesetzt sowie als Film dargeboten werden (Barone und Eisner 2012; Chamberlain et al. 2018; Jones et al. 2008; Knowles und Cole 2008; Leavy 2017). Wenn auch anzumerken bleibt, dass sich zu möglichen einzelnen - in einer Ausstellung konvergierenden - Elementen Auseinandersetzungen finden, so eben zur »bildenden Kunst « (Malerei, Bildhauerei), zu (Video-)Installationen, Fotografie etc.

\section{Annotationen zur performativen Sozialwissenschaft}

Den Gegenstandsbereich und die Potenziale der performativen Sozialwissenschaft skizziert Kip Jones $(2017,3)$, einer der renommiertesten Vertreter, wie folgt, wobei auch bei ihm Ausstellungen unerwähnt bleiben:

»Performative Social Science embraces the use of tools from the Arts (e. g., photography, dance, drama, filmmaking, poetry, fiction, etc.) by expanding - even replacing - shop- 
worn methods of research and diffusion of academic efforts. A [...] potential of these new Arts-based methods of exploration and dissemination is inclusion in these processes of the very communities that we research and/or try to reach with our investigations. When all three elements (Research/Dissemination/Community) are based in an Arts-based approach and are working in tandem, Performative Social Science is at its best. «

Dass performative Sozialwissenschaft eine hohe Affinität zur qualitativen Forschung aufweist und sich innerhalb dieses Feldes ausbreiten und (weiter-)entwickeln konnte, hat verschiedene Gründe: Ganz im Einklang mit Grundüberzeugungen der - auch von den Literaturwissenschaften inspirierten - qualitativen Forschung wird die Rezeption von wissenschaftlichen Ergebnissen als interpretativer Akt verstanden (Ricœur 1981; Straub 1999). Allerdings wird innerhalb der performativen Sozialwissenschaft immer wieder auf die Begrenztheit von traditionellen Ergebnisdarstellungen in Texten (Artikeln, Berichten etc.) hingewiesen (Gergen und Gergen 2010). Die Fixierung auf diese Präsentationform stelle gleich mehrfach eine Einschränkung dar: Zum einen würden nicht alle Sinne angesprochen, zum anderen werde mit der Monokultur wissenschaftssprachlicher Darstellung eine vom » traditionellen « Wissenschaftssystem geforderte Klarheit (Eindeutigkeit) und Exaktheit unhinterfragt präjudiziert. Da eine solche Haltung die Annahme der Unabschließbarkeit von Deutungen unterlaufe, favorisieren performative Ansätze eine Darstellung, die Mehrdeutigkeiten eröffnet und den Rezipierenden eigene Interpretationsspielräume bietet. Zusätzlich zu der innerwissenschaftlichen Kritik kommen außerwissenschaftliche Argumente hinzu: Insbesondere wird diagnostiziert, dass sich im Wissenschaftssystem eine eigene Sprache durchgesetzt hat, die für interessierte Lai*innen oder Angehörige anderer Fachdisziplinen wenig zugänglich ist; eine Forderung, die aber zunehmend lauter wird im Zuge der Open Society und einem Verständnis von »Wissenschaft als öffentliches Gut « (Mruck, Gradmann und Mey 2004). Insofern meint dieser Diskurs nicht nur eine veränderte Disseminationsstrategie, sondern dass Forschungsarbeiten den (beforschten) Akteur*innen Handlungsoptionen offerieren und in diesem Sinne »interventionistisch « sein sollten (Winter 2010).

Es überrascht nicht, dass es nicht den einen Präsentationsmodus »Ausstellung « gibt angesichts der möglichen Bandbreite an Ausstellungskonzepten - wie die ausschließliche Präsentation von Objekten/Artefakten (im Kontext von wissenschaftlichen Ausstellungen auch zu übersetzen als die »Ergebnisse « einer Studie); die Ermöglichung zur Raumerkundung und Interaktion mit Exponaten (lies: Ergebnispräsentationen) bis hin zu virtuellen Darbietungen (in der Ausstellung selbst oder als reine Internetpräsentation, oft als »Ausstellungsrundgang « angelegt). Zudem ist bei den diversen Formen der Verknüpfung von Kunst mit Wissenschaft im Rahmen der performativen Sozialwissenschaft zu unterscheiden, ob - wie bei dem Ansatz der Arts-informed Research die künstlerischen Darstellungsformen primär genutzt werden, um die Ergebnisse von 
Forschung zu vermitteln, oder ob die künstlerischen Praktiken auch zur Erkundung von Phänomenen genutzt werden, wie es mittels der in den Kunstwissenschaften verankerten Artistic Research - und daran angelehnt bei der sozialwissenschaftliche Variante der Arts-based Research - praktiziert wird (Schreier 2017).

Im Folgenden werde ich zunächst drei von mir realisierte Ausstellungen kurz vorstellen (Abschnitt 3.1) und dann einige weitere Ausstellungen skizzieren (3.2), um so generelle Muster für das Präsentationsformat »Ausstellung « zu konturieren. Im anschließenden vierten Abschnitt wird dann zusammengetragen, in welcher Weise Interviewdaten in solche Präsentationsformate Eingang finden (4.2) und wie sich entlang der Ansprüche von Arts-informed Research und Arts-based Research Studienergebnisse darstellen lassen (4.3); eingeleitet werden die Überlegungen mit Anmerkungen zur Forschungsethik (4.1). Abgeschlossen werden diese Darlegungen mit der Frage nach der möglichen Bewertung solcher Arbeiten und jener, inwieweit hier Aspekte der Geltungsbegründungen qualitativer Forschung heranzuziehen oder andere Gütekriterien zu entwickeln sind (5.).

Der Fokus meiner Ausführungen begründet sich damit, dass qualitative Forschung ungeachtet ihrer Weiterentwicklung und der Diversität an Zugängen nach wie vor vornehmlich als Interviewstudien angelegt wird (Breuer et al. 2014, 262-70). Dass ich ausschließlich auf qualitative Forschungsarbeiten zentriere, reflektiert wiederum die Nähe zwischen performativer Sozialwissenschaft und qualitativer Forschung, wobei hier eine Akzentuierung auf die Frage der Geltungsbegründung vorgenommen wird, da bislang sehr wenige explizite Ausführungen vorliegen, wie denn die Integration empirisch-qualitativer Daten in performativ umgesetzte Präsentationen im Rahmen von Ausstellungen vollzogen werden kann.

\section{Ausstelllungen: Beispielhafte Umsetzungen eines Formats}

\subsection{Eigene Ausstellungen}

In den zurückliegenden Jahren habe ich hervorgehend aus Forschungsstudien zu den Themen Angst, Heimat und DDR-Jugendkultur Ausstellungen realisiert: Während 2011 die Ausstellung zum Erleben und Umgang mit Angst im Rahmen des elftägigen »Angst(frei)-Festivals « im öffentlichen Stadtraum und in der ehemaligen JVA von Stendal gezeigt wurde, wurden 2014 die Ergebnisse einer Studie zu Heimatkonstruktionen als »Skulpturale Collagen « im Rahmen des »Heimatperspektiven «-Events an einem Wochenende in einem leerstehenden Stendaler Kaufhaus als Ausstellung gestaltet. Die Studie »Jugendkultur in Stendal: 1950-1990« ist 2018 als viermonatige Präsentation im Altmärkischen Museum plus kulturellen und wissenschaftlichen Begleitprogramm umgesetzt worden. 


\section{Anmerkungen zu den den Ausstellungen zugrunde liegenden Studien}

Im Aufbau weisen die den Ausstellungen vorausgehenden qualitativ-empirischen Studien eine ähnliche Struktur und einen vergleichbaren Ablauf auf: Sie sind als regionalbezogene Forschungsprojekte angelegt; ihre Umsetzung wurde zunächst im Rahmen von Lehrforschungsprojekten initiiert und dann mit einem kleinen Forschungsteam und bis zur Umsetzung kooperativ mit Kunst- und Kulturschaffenden weiter vorangetrieben. In allen Studien wurden nach Vorstellung des allgemeinen Rahmenthemas zunächst die Forschungsfrage und dazugehörige Untersuchungsfragen präzisiert (Gläser und Laudel 2010, 60-73) und darauf aufbauend die Interviewleitfäden - unter Bezug auf die Vorschläge zur Leitfadenkonstruktion von Helfferich (2011, 178-89) - entwickelt; die Informationsblätter und Interviewvereinbarungen entworfen (inklusive einer $\mathrm{Zu}$ satzabrede, die Daten öffentlich präsentieren zu können; siehe Abschnitt 4.1) und die Akquise potenzieller Interviewter eingeleitet. Die Gespräche - zumeist als problemzentrierte Interviews (Witzel 2000; Witzel und Reiter 2012; vgl. Mey 2000) - wurden je nach Projektziel entweder per Audio oder Video aufgezeichnet. Die Auswertung sah vor, dass zunächst eine Globalanalyse (Legewie 1994) geleistet wurde, mit der eine thematische Ordnung inklusive eines Inhaltsverzeichnisses und eine Auswahl einschlägiger Zitate kollektiert sowie eine nachzeichnende Zusammenfassung erstellt und ein Motto als charakteristische Gesamtaussage zum Interview vergeben wurden; darauf aufbauend erfolgte - begleitet von zusätzlicher Transkription der Interviews oder Teilverschriftlichung relevanter Passagen in Form der literarischen Umschrift als eine Variante der erweiterten inhaltlich-semantischen Transkription (Dresing und Pehl 2018, 20-26) eine thematische Kodierung in Anlehnung an die Grounded-Theory-Methodologie (Mey und Mruck 2009), um kategoriale Bestimmungen vornehmen zu können. Nach Abschluss der eigentlichen Forschungsarbeit wurde die Aufbereitung der Ergebnisse für die öffentliche Präsentation geleistet. Neben der Skizzierung der Ausstellungsarchitektur waren dies die - fortgesetzte und weitergehende - Recherche nach Objekten und Dokumenten, die Erstellung von Installationen sowie die Aufbereitung (Zusammenschnitt) von Audio- beziehungsweise Videofiles. Das jeweilige Ausstellungskonzept wurde immer parallel ausgearbeitet, zu Beginn der Studie bestand lediglich die Grundidee.

\section{Ausstellung »Angst(frei)«}

Im Rahmen des $\gg$ Angst(frei)-Festivals « - organisiert vom Theater der Altmark ${ }^{2}$ wurden die Ergebnisse der Projektstudie auf zwei Wegen präsentiert. Zum einen wurden im Stadtraum insgesamt 36 großformatige Porträtaufnahmen der Interviewten mit einem aussagekräftigen Zitat versehen: Angst sollte so ein »Gesicht « bekommen und »öffentlich « gemacht werden (Abb. 1). 


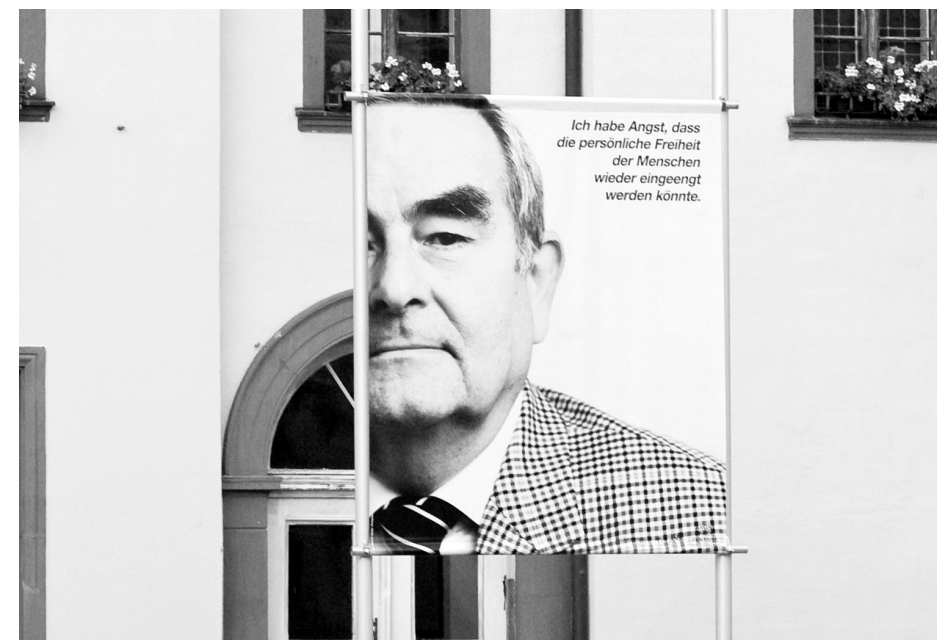

Abbildung 1: Im öffentlichen Raum platzierte, großformatige Porträtaufnahme mit einem ausgewählten Interviewzitat; »Angst(frei)-Festival«, Hansestadt Stendal 2011 (Foto: Sascha Löschner).

Zum anderen wurden auf dem Ausstellungsgelände, die ehemalige JVA, die einzelnen Zellen als Ausstellungsräume für diverse Arbeiten verschiedener Beteiligter genutzt. Das von mir geleitete Teilprojekt präsentierte sich in einem mehrräumigen Zellentrakt. Im Entréebereich lief in Endlosschleife eine Zitatencollage, montiert aus Auszügen von den Projektsitzungen - insbesondere Konzeption der Studie und Auswertungssitzungen - und eingelesenen Passagen aus Reflexionsmemos der Projektmitarbeitenden; diese explizite Referenz sollte die Bezogen- und Betroffenheit kenntlich machen, indem auch die Forschenden selbst sich zum Thema machen (Mruck und Mey 1998). Im zweiten Raum liefen Videos mit »Experten« zum Thema Angst aus psychologischer Perspektive. Im dritten und vierten Raum befanden sich eine Hörstation mit allen Originalinterviews im Audioformat und ein Regal mit aktenähnlichen »Dossiers « (Abb. 2). In diesen waren die Interviews auf Einzelfallebene aufbereitet, dazu gehörten ein Motto, eine zweiseitige Zusammenfassung, ausgewählte Zitate und ein Inhaltsverzeichnis mit Zeitmarken, sodass die Originalaufnahmen auf der Hörstation gezielt angesteuert werden konnten.

Der fünfte Raum war nicht zugänglich, sondern durch eine Scheibe abgetrennt, hinter der ein Monitor stand, wobei das dort gezeigte Videointerview nicht hörbar war. Leitend war hierbei die Idee, dass die Besuchenden bei dem Versuch, zu erahnen, worüber der Interviewpartner spricht, auf ihre eigenen Emotionen und Erlebnisse zum Thema Angst verwiesen werden. Auf dem Boden aller Räume lagen auf Folien 


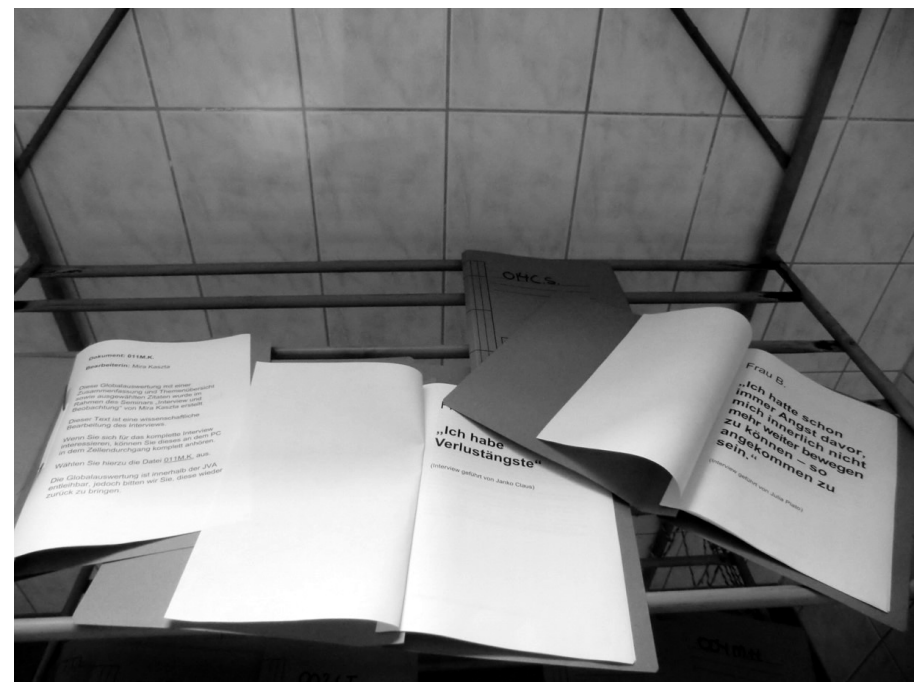

Abbildung 2: Dossiers (Schnellhefter) mit ausgewähltem Zitat (»Motto«) auf dem Deckblatt und aufbereiteten Informationen (Inhaltsverzeichnis, Zusammenfassung und ausgewählte Zitate) aus den Interviews; »Angst(frei)-Festival«, Hansestadt Stendal 2011 (Foto: Sascha Löschner).

kopierte »Angst-Aussagen «, welche über die Dauer der Ausstellung mehr und mehr Nutzungspuren aufwiesen, sodass die Angst also sozusagen »verblasste « (Mey 2011).

Insgesamt bot das elftägige Festival eine Fülle an Veranstaltungselementen aus Workshops, Lesungen, Theateraufführungen, Podiumsdiskussionen, Führungen und Konzerten (Theater der Altmark 2011). Die öffentliche Thematisierung im Rahmen des »Angst(frei)-Festivals « hatte gleich zwei relevante Ausgangspunkte: Zum einen lag der Anschlag auf das World Trade Center zehn Jahre zurück, infolgedessen ein neuer Sicherheitsdiskurs (inklusive aller erdenklichen Formen von Überwachungsmaßnahmen, die ihrerseits Angst erzeugen) einsetzte; zum anderen wurde ein Befund einer deutschen Krankenversicherung aufgegriffen, nachdem die Menschen in Sachsen-Anhalt, statistisch gesehen, » am ängstlichsten « seien. Die Wahl eines »Gefängnisses « als Ausstellungsort und die im öffentlichen Raum ausgestellten AngstAussagen reflektierten dieses Spannungsverhältnis; darüber hinaus war sowohl der psychologische Aspekt (Angst als individuell erlebtes als auch verhandeltes und oft verschwiegenes Thema) sowie die gesellschaftliche Rahmung (Schutzmaßnahmen) bei der Realisation leitend und auch die grundsätzliche Idee bei der Gestaltung des Zellentraktes. 


\section{Ausstellung »Skulpturale Collagen«}

Im Rahmen des »Heimatperspektiven «-Events - als Gemeinschaftsprojekt mit dem Theater der Altmark ${ }^{3}$ - wurden die Interviews (geführt mit Menschen verschiedenen Alters und aus verschiedenen Regionen) überwiegend als Installationen (»Skulpturale Collagen «) umgesetzt und auf Basis der Ergebnisse Objekte und Raumangebote auf der unteren Ebene eines leerstehenden Kaufhauses in der Einkaufsstraße von Stendal arrangiert. So waren Steine, die eine Mauer bildeten, mit gehaltvollen Worten beschriftet (Abb. 3); Zitatauszüge auf eine Gruppe von Schaufensterpuppen montiert (Abb. 4); eine Sitzreihe mit verschiedenen Stühlen und davor Interviewzitate ausgelegt (Abb. 5); begehbare Räume mit auf Spiegel lesbaren Zitaten oder eine Sitzstation mit Audiosamples eines Interviews gestaltet. Auch Installationen ohne Zitate symbolisierten die Auseinandersetzung mit Heimatperspektiven - eine Gruppe mit TV-Geräten (Abb. 4), auf denen unter anderem deutsche Heimatfilme liefen (die ursprünglich in nicht deutscher Sprache über Kopfhörer abrufbar sein sollten); eine artifizielle $\mathrm{Zu}$ sammenstellung von Speermüll (Abb. 6) oder ein Schredder, der »Heimatromane « zerschnitt.

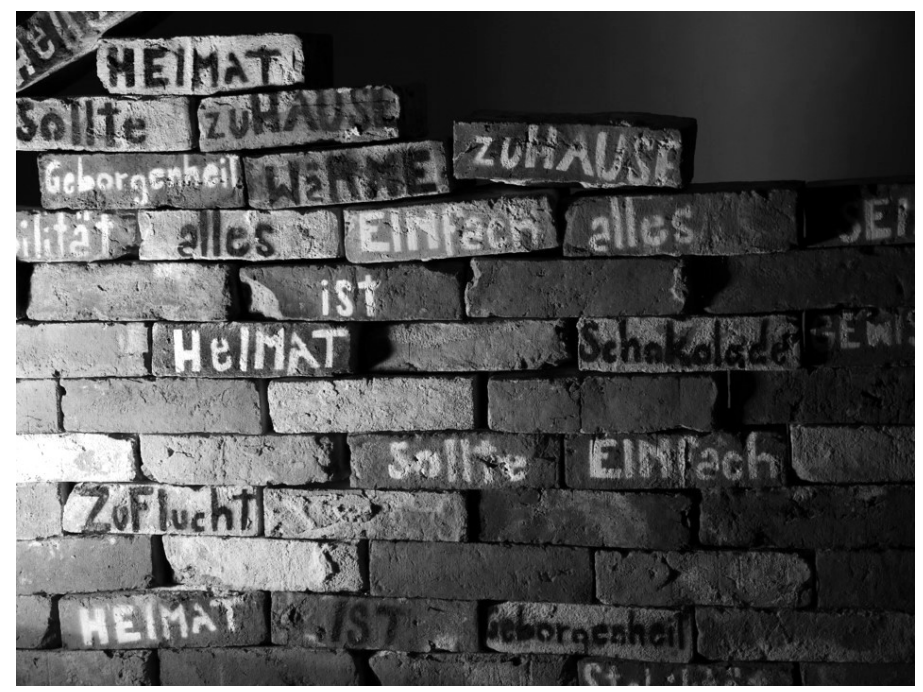

Abbildung 3: Zitatekollektionen übertragen auf Mauersteine; Installation »Sicherheit« von Christine Hunger, Josephine Kirchner, David Lenard, Günter Mey; Skulpturale Collagen, Heimatperspektiven, Hansestadt Stendal 2014 (Foto: Susanne Moritz). 


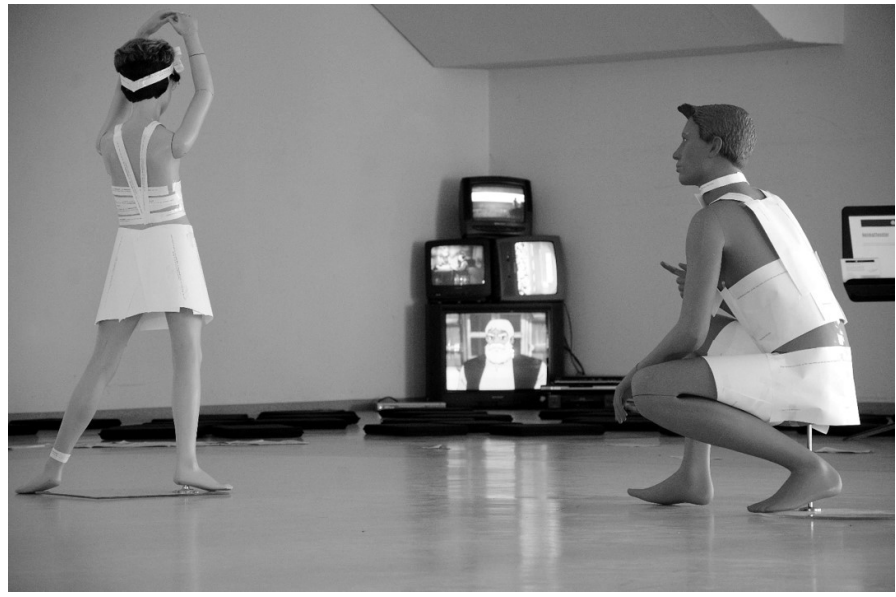

Abbildung 4: Zitate montiert auf Schaufensterpuppen; Installation »Identität« von Christine Hunger, Josephine Kirchner, David Lenard, Günter Mey; im Hintergrund Videoinstallation; Skulpturale Collagen, Heimatperspektiven, Hansestadt Stendal 2014 (Foto: Susanne Moritz).

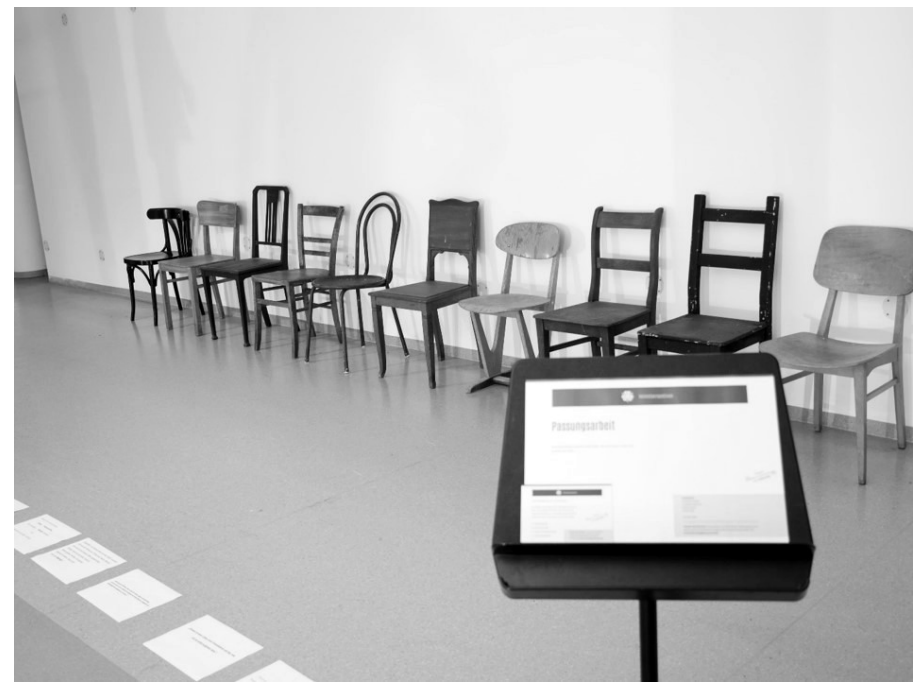

Abbildung 5: Interviewauszüge vor aufgereihten Stühlen; Installation »Passungsarbeit« von Christine Hunger, Josephine Kirchner, David Lenard, Günter Mey; Skulpturale Collagen, Heimatperspektiven, Hansestadt Stendal 2014 (Foto: Susanne Moritz). 


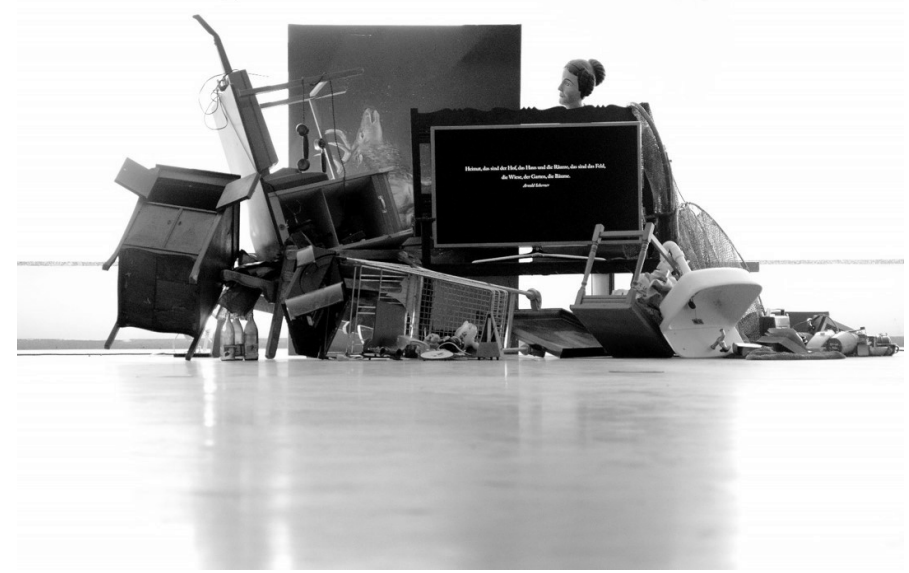

Abbildung 6: Installation »Heimatschrott« von Christine Hunger, Josephine Kirchner, David Lenard, Günter Mey; Skulpturale Collagen, Heimatperspektiven, Hansestadt 2014 (Foto: Susanne Moritz).

Gerahmt wurde das Event durch Musik- und Freizeitangebote auf der abgesperrten Straße vor dem Gebäude. Die Ausstellung war als temporäres Event geplant und wurde danach abgebaut. Ausgelegt - und damit » konservierbar « - wurden lediglich vier Mitnehmposter mit konträren »Heimataussagen «: » Heimat ist in mir «, »home is where my heart is «, »Heimat - in der Welt zu Hause « und »Heimat ist dort, wo ich (als Mensch) sein kann/darf «. Angezeigt wurden damit die wesentlichen Konstruktionen und das Hauptergebnis des $\gg$ Sich-Be-Heimat-en « als Prozess und darin eingelagerter identitärer Momente - von Differenzerfahrung und Passungsarbeit - inklusive der Fragen nach Anerkennung und Zugehörigkeit.

\section{Ausstellung »Jugendkultur in Stendal: 1950-1990«}

Anders als diese Präsentationen im öffentlichen Raum oder die temporäre Nutzung von Orten war die Ausstellung »Jugendkultur in Stendal: 1950-1990 « museal konzipiert (Mey 2018b). Im Sonderausstellungsbereich des Altmärkischen Museums wurden mit »der sound «, »der style « und »die events « drei Themenräume gestaltet, um Perspektiven auf jugendkulturelle Praxen jenseits - vergleichsweise häufig erforschter urbaner Milieus zugänglich zu machen (Leser und Mey 2017). Jeder Raum hatte neben Fotos, Magazinen, Objekten eine zentrale Installation: Im ersten Raum zu »der sound « war es eine raumteilende Hängung mit Schallplattencovern, die flankiert wurden von einem Schallplattenregal und Schallplatten auf dem Boden; in dem zweiten 


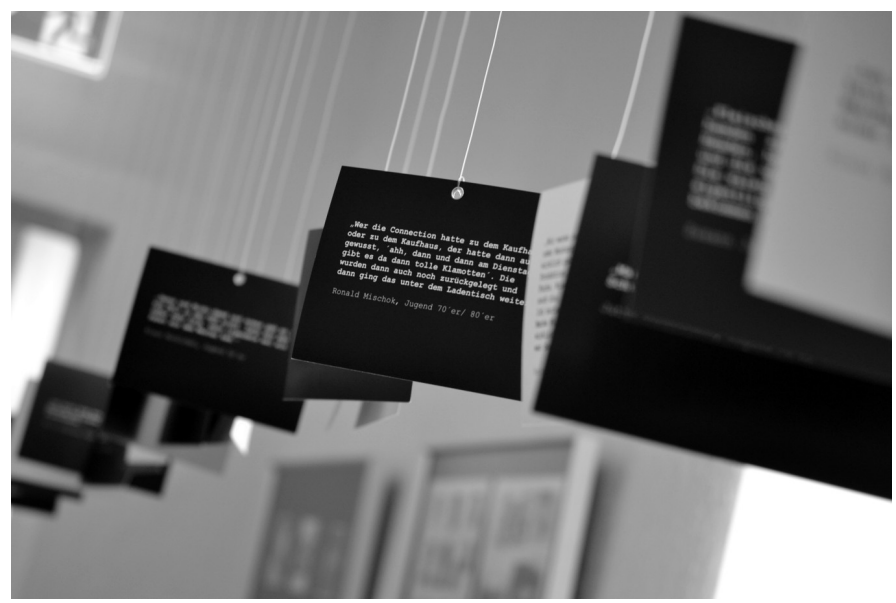

Abbildung 7: Zitate auf Postkarten als Fluchtlinie durch alle Ausstellungsräume aufgespannt; Ausstellung »Jugendkultur in Stendal: 1950-1990«, Hansestadt Stendal 2018 (Foto: Luisa Simon).

Raum zu »der style « wurde eine an der Wand auf montierte Büsten getroffene Auswahl von Kleidung sowie ein Kleiderständer und Koffer arrangiert; der dritte Raum hatte eine Fototapete eines alten Lokals mit original dazu passenden Sesseln davor sowie eine mit Gucklöchern versehene Vitrine, durch die die diversen Lokale und Treffpunkte auf einer Stadtkarte zu sehen waren. In dem Vorraum (Entrée) wurde über eine Diashow mit Bildern aus vier Jahrzehnten in das Thema eingeführt. Die Interviews wurden auf verschiedenen Kanälen eingespeist: Über die gesamte Ausstellungsfläche - vom Vorraum über die drei Themenräume - bildeten auf Postkarten gedruckte Zitate eine - greifbare - Fluchtlinie auf Augenhöhe (Abb. 7). Im Vorraum befand sich ein Poster-Blättergestell mit 30 Kurzporträts der 45-80-jährigen Interviewten (Abb. 8). Die Texte enthielten biografische Notizen zu Musik, Kleidung und Events. In jedem Raum liefen auf Monitoren jeweils circa 30-minütige, aus den Interviews erstellte Videocollagen zu dem jeweiligen Thema (Abb. 9). ${ }^{4}$

Flankiert wurde die viermonatige Ausstellung durch ein kulturelles und wissenschaftliches Begleitprogramm - mit Filmen, Theater, Lesungen sowie Erzählcafés ${ }^{5}$ und einer Fachtagung -, das als Gestaltungsprinzip hatte, sich jeweils nacheinander pro Monat einem Jahrzehnt zu widmen. Schließlich wurde im Nachgang der Ausstellung noch eine Webseite ${ }^{6}$ entwickelt, in der die Ausstellungsarchitektur virtuell übersetzt wurde. Als Leitlinie für die Gesamtpräsentation galt es, das über die vier beforschten Jahrzehnte rekonstruierte zentrale Thema des Changierens zwischen der » verordneten « DDR-Offizial- und der eigenproduzierten » alternativen « Nischen- 


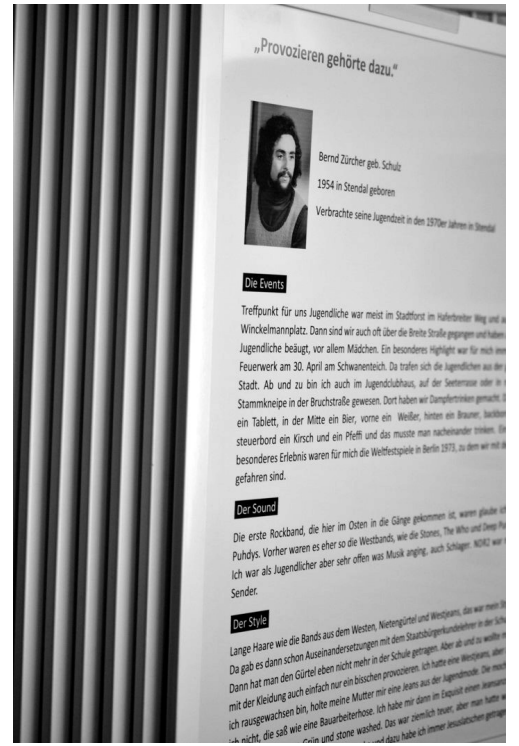

Abbildung 8: Aus den Interviews aufbereitete Kurzporträts in einem Poster-Blättergestell; Ausstellung »Jugendkultur in Stendal: 1950-1990«, Hansestadt Stendal 2018 (Foto: Luisa Simon).

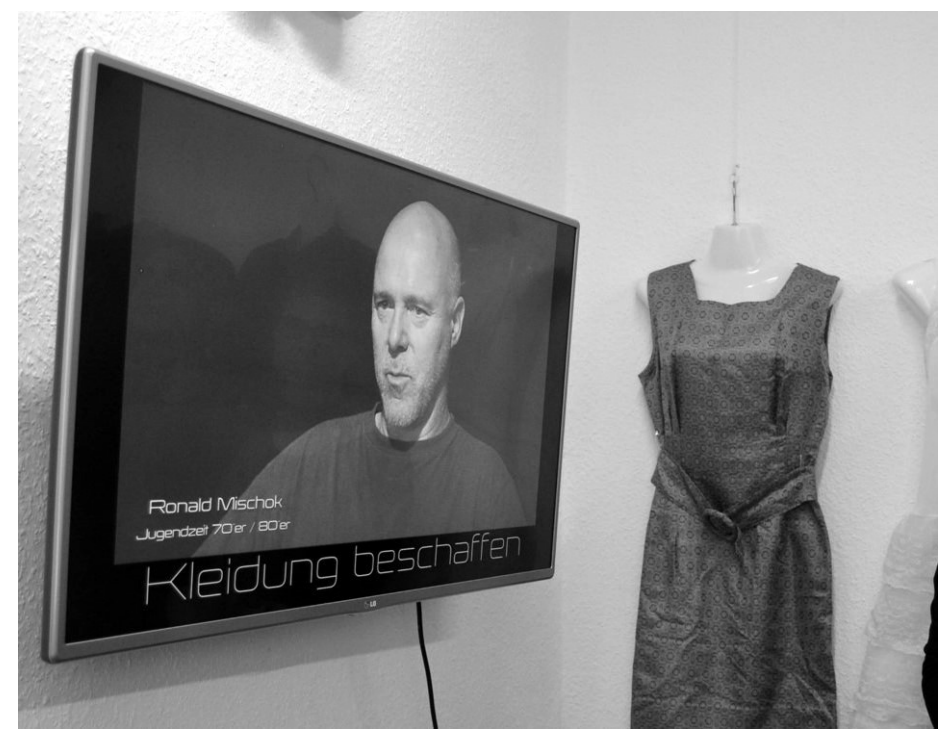

Abbildung 9: Interviewcollage auf Bildschirm in einer Endlosschlaufe; Ausstellung »Jugendkultur in Stendal: 1950-1990«, Hansestadt Stendal 2018 (Foto: Luisa Simon). 
kultur inklusive der darin erkennbaren (An-)Passungsprozesse nachzuzeichnen, wobei als besonders markant die Westorientierung via Kleidung und Musik und die damit antizipierbare Provokation durch den Rekurs auf den »Klassenfeind « als Interpretationsfolie angeboten wurde.

\subsection{Generelle konzeptionelle Umsetzungen von Ausstellungen}

Bei der Konzeption der dargestellten Ausstellungsumsetzungen sind verschiedene Stilmittel und Präsentationsformen eingegangen, die sich auch im Kontext anderer Ausstellungen finden und sich zunehmend als wiederkehrende Elemente ausmachen lassen, wenn Studien auf diese Weise öffentlich gemacht werden. Erkennbar bei meinen Realisierungen sind Analogien zur sogenannten »Bürgerausstellung « (Böhm, Legewie und Dienel 2008; Keppler, Böhm und Dienel 2013), die insbesondere in den Arbeiten aus dem Umfeld von Heiner Legewie genutzt wurde. Hierbei werden die Ergebnisse aus Studien (überwiegend basieren sie auf Interviews) in Form von Ergebnispostern aufbereitet, in denen zumeist fallorientiert die zentralen Auszüge aus den Interviews zusammengefasst, besonders prägnante Zitate ausgewählt und mit Fotos der Befragten versehen werden. Die so aufbereiteten Poster sind als Ausstellung oder als Rundgang aufgebaut, häufig in für die Bürger*innen zentralen Einrichtungen. Die Ausstellungseröffnung wird - wenn möglich auch partizipativ - unter Einbezug der Befragten gestaltet, die auch auf diese Weise zu Wort kommen sollen.

Eine der ersten Arbeiten von Legewie (2003) wurde im Rahmen einer gemeindepsychologischen Studie umgesetzt, in der er die Auswirkungen der Wandlungsprozesse insbesondere des Massentourismus und der ökonomischen Aufwertung historischer Wohnquartiere in Berlin und Florenz untersuchte. Hierbei erhob er neben Erfahrungen und Wünschen der Befragten auch deren konkrete Verbesserungsvorschläge für die Lebensbedingungen in ihrem Viertel. Mit der öffentlichen Präsentation als »Bürgerausstellung « in beiden Städten - und den in dem Begleitkatalog in italienischer und deutscher Sprache erstellten Bewohner*innenporträts - verband Legewie das Ziel, den vielfältigen Sichtweisen von Bürger*innen und Expert*innen ein Forum zu geben und damit (Denk-)Anstöße für den öffentlichen Diskurs über aktuelle Probleme urbaner Lebensqualität anzuregen.

Solche über aufbereitete Poster angelegte Ausstellungen werden auch wiederkehrend im Berliner Museum Europäischer Kulturen umgesetzt, so etwa zu »Europas neue Alte « (Kostas und Ziehe 2016), die als foto-ethnografische Ausstellung realisiert wurde, in deren Rahmen 27 »Fotoessays « von Personen im Rentenalter aus unterschiedlichen Gesellschaftsschichten und aus dem urbanen sowie dem ländlichen Raum porträtiert wurden. Ebenfalls als foto-ethnografische Ausstellung realisiert wurde »I'm 
not afraid of anything « (Ziehe und Zippel 2013), um junge Menschen aus Europa zu porträtieren. In dieser Ausstellung wurden auch filmische Interviews einer Projektgruppe der Humboldt-Universität zu Berlin integriert und an Terminals konnten Daten der schriftlichen Befragung hinsichtlich verschiedener Fragestellungen eingesehen und interaktiv ausgewertet werden.

Mittlerweile sind die Porträts - ob als Poster oder Fotoessay bezeichnet - eine recht gängige Form, Ergebnisse einfach zugänglich zu machen. Was noch vor 20 Jahren als eine recht neue Form der Präsentation für die Öffentlichkeit galt, ist mittlerweile ein im öffentlichen Raum - etwa auf zentralen Plätzen oder in Bahnhöfen - häufig vorfindliches Format. Hier gilt zwar ein Höchstmaß an Dissemination, aber auch eine wenig künstlerisch herausfordernde Umsetzung mit Blick auf Layout, Design und Fotos; auch wenn Letztere in vielen Projekten von professionellen Fotograf*innen erstellt wurden.

Zunehmend wird auch die Verwendung von Audio-/Videomaterial, abrufbar direkt über Hörstationen (teilweise via App anwählbar), genutzt und weiterführende Informationen visuell aufbereitet. So wurden bei der im Kurpfälzischen Museum gezeigten Ausstellung »Eine Stadt bricht auf - Heidelbergs wilde 70er « ausgehend von dem lokalspezifischen Ereignis - die geplanten Sanierungsmaßnahmen der Altstadt die sich in den 1970er Jahren herausbildende »alternative Szene « (Studentenbewegung, Anti-AKW- und Frauenbewegung, die Etablierung von Wohngemeinschaften etc.) in Heidelberg beleuchtet. In Ausstellungsräumen wurden Filme, Dokumente, Fotografien und Objekte präsentiert. Mit zusätzlichen Hintergrundinformationen und Interviewauszügen wurde ein umfassender Eindruck der damaligen Aufbruchstimmung und Protestkultur gegeben. Die Ausstellung basierte - initiiert von Manfred Metzner, Verleger des Wunderhorn-Verlags - auf einem gemeinsamen Studienprojekt »Das alternative Milieu der 60er und 70er Jahre « am Historischen Seminar sowie dem Institut für Europäische Kunstgeschichte der Universität Heidelberg unter der Leitung von Cord Arendes und Henry Keazor. Ein besonderes Detail war, dass von dem Projektteam die Informationen zur Forschungsarbeit und Ausstellung im Stile der » alternativen Stadtzeitungen « - die sich als »Gegenöffentlichkeit « in fast allen (Studierenden-)Städten gründeten - aufbereitet wurden.

Der Einbezug von künstlerischen Exponaten - und deren Konfrontation mit aufbereiteten Interviewplakaten - findet sich, passend zum Sujet der Studie, bei Legewie, Eva Jaeggi und Jarg Bergold. Hier wurden die Ergebnisse ihrer Studie zu »Kreativität im Alter «, bei der ältere Künstler*innen interviewt wurden, um deren Werke ergänzt (http://schoepferisch-im-alter.blogspot.de/). Dieses Format nutzte Legewie ebenso bei der Präsentation seiner Studie »Künstler in Athen - Stadt der Krise « (Legewie und Eichinger 2017). In dieser wurden griechische Künstler*innen interviewt und bei ihrer Arbeit fotografiert, um aufzuzeigen, wie Kunst, Künstler*innenbiografie 
und die in Griechenland vorherrschende ökonomische Krise miteinander in Beziehung stehen. ${ }^{7}$

Eine deutliche von Exponaten und Installationen dominierte Ausstellung stellt »Megacool 4.0 - Jugend und Kunst « (Richard und Krüger 2012) dar, die darauf abzielte, Jugendkultur mit den Mitteln zeitgenössischer Kunst darzustellen. Gezeigt wurden Fotografien, Medien- und Videokunst, Malerei, Streetart und Skulpturen verschiedenster internationaler Künstler*innen. Darüber hinaus wurden Objekte und Alltagsgegenstände aus diversen Jugendszenen präsentiert. Zudem wurden Exponate von jugendkulturell »typischen « Stilen (u. a. Gothics, Hipster, Hip-Hopper, Metalheads, Raver etc.) per interaktiver Installationen visualisiert.

Von Künstler*innen und Sozialwissenschaftler*innen gemeinsam kuratierte Ausstellungen gehen auf die Zusammenarbeit von Bjarne Sode Funch von der Roskilde Universität und Inge Merete Kjeldgaard vom Esbjerg Kunstmuseum zurück. Unter dem Motto »Art in Context « organisierten sie vier Ausstellungen in Dänemark, wobei sie für jede jeweils eine*n Wissenschaftler*in und eine*n Künstler*in zusammenbrachten, um das jeweilige Thema umzusetzen. Dies waren: 2007 An Sigt « (»Ein Ziel «), 2008 $\gg$ The Map is not the Territority«, $2011 »$ Live Tegn « (»Lebenszeichen «) sowie 2013 »What I am doing here? An Exhibition on Art and Existence « (Funch 2015).

\section{Interviews ausstellen}

\subsection{Annotationen zur Forschungsbeteiligung und -ethik}

Die vorgestellten Ausstellungen weisen bei aller Diversität auf ein mögliches Spektrum hin, wie Ergebnisse aus Interviewstudien aufbereitet präsentiert werden können. Im Folgenden werden einzelne Umsetzungen - so die Verwendung als Rohmaterial, in Form von aufbereiteten Porträts sowie von Zitatauszügen und die Erstellung von Videokompilationen - kommentiert.

Um all diese Umsetzungen zu realisieren, ist wichtig voranzustellen, dass eine Einwilligung der Interviewten vorliegt, das Originalmaterial öffentlich präsentieren zu dürfen. Daher wurden in meinen Projekten die schriftlichen Interviewvereinbarungen, die die datenschutzrechtlichen Belange (so auch zur Anonymisierung und Verwertung) regeln, mit entsprechenden Zusatzabreden versehen: Für gewöhnlich biete ich dafür die Optionen, dass das Material (vollständig oder in Auszügen ohne oder mit Verfremdung) sowie rein auditiv oder visuell für öffentliche Präsentationen benutzt werden darf. So besteht zumindest auch bei der Wahl einer anonymisierten Variante die Möglichkeit, die Interviews technisch bearbeitet (Veränderung der Stimme oder auch nachträgliches Einlesen des Transkripts) zu berücksichtigen. Ungeachtet dessen 
bleibt aber immer bei dem Wunsch nach Anonymität abzuwägen, gänzlich auf das Material zu verzichten, da - selbst wenn Klarnamen von Personen und Einrichtungen oder Orten durch ein Störgeräusch/Schwärzung unkenntlich gemacht werden aufgrund dargestellter Handlungsabläufe, bestimmter Ereignisfolgen oder spezifischer Sprachmerkmale (Redewendungen, Ausdrücke etc.) Rückschlüsse auf die Person möglich sind.

Vor diesem Hintergrund - und sicher insbesondere dann, wenn Darstellungen mit Klarnamen oder zusätzlich mit Fotomaterial vorgesehen sind, und damit eine eindeutige Kenntlichmachung der Person vorliegt - ist eine Einbindung der Interviewten im Sinne einer »kommunikativen Validierung « (member checking; Flick 2017) anzustreben, sodass sie sich »richtig « verstanden wissen. Dieses partizipative Vorgehen bedeutet letztlich auch, Anmerkungen, Ergänzungen, Korrekturen aufzugreifen oder zumindest in einen (mitunter langwierigen) Dialog zu treten und Perspektiven auszuhandeln (von Unger 2017).

\subsection{Vom Rohmaterial bis zur Poetic Transcription: Zum Umgang mit Interviewmaterial}

Das möglicherweise »einfachste « Vorgehen stellt die Nutzung des Rohmaterials dar. Die aufgezeichneten Interviews werden unkommentiert und unbearbeitet dargeboten, bis auf technische Nachbearbeitungen, wie im Falle von Audio-/Videofiles durch Einsatz von Ton-/Farbfiltern etc.; bei Transkripten etwa die Fehlerkorrektur und Notationsanpassung. Dieses Bereitstellen von (anonymisierten) Originaldaten entsprach in der qualitativen Forschung lange Zeit der Dokumentationspraxis zur Transparenzherstellung im Rahmen von Qualifikationsarbeiten, wobei die in Anhängen versammelten Transkripte nicht öffentlich zugänglich waren.

Die Aufbereitung der Ergebnisse in Dossiers oder Fallporträts stellt eine Präsentationsform dar, die nahe an traditionellen Darstellungen qualitativer Forschungsstudien ist; allerdings wird/werden die (Fall-)» Geschichte(n) « im Kontext der Ausstellungen (und den Begleitpublikationen) möglichst einfach gehalten, um sie schnell lesbar und (alltags-)verständlich zugänglich zu machen. In der Regel unterliegen diesen Darstellungen die in den Auswertungen herausgearbeiteten Kategorien, ohne diese allerdings explizit auszuweisen; sie lassen sich vielmehr durch die biografischen Verdichtungen oder szenischen Episoden erahnen. Die Verwendung einzelner Interviewauszüge - als »Motto « oder »Zitat « - ist eine sehr prägnante Vorgehensweise, mit der einzelne Aspekte herausgehoben oder aber Haltungen zu und Erfahrungen mit einem Thema generell » auf den Punkt « gebracht werden können. Die Zitate werden dabei oft stilistisch bearbeitet, indem parasprachliche Merkmale eliminiert beziehungsweise die 
Aussagen in eine Standardorthografie gebracht werden. Darüber hinausgehend lassen sich die Passagen im Rahmen einer »Poetic Transcription « in eine literarische Gattung überführen (Faulkner 2009; Johnson et al. 2017; Prendergast, Leggo und Sameshima 2009). Generell gilt bei der Bearbeitung von Aussagen, dass diese von den Personen, die sie getätigt haben, » abgehoben « werden können, was den Allgemeinheitsanspruch unterstreicht. Die Verknüpfung mit dem (Klar-)Namen dagegen markiert eher die persönliche Positionierung und über die Kollektion diverser Zitate lässt sich die mögliche Bandbreite inklusive der Gegenpositionen »in den Raum « stellen.

Collagen aus den Interviews (ob als Skript oder als Video), in denen Auszüge aus verschiedenen Interviews » miteinander ins Gespräch « gebracht werden, eignen sich besonders, um über verschiedene Personen hinweg eine Storyline zu entwickeln, bei der sozusagen »das eine Wort das andere gibt«. Aber ebenso können über die Montage Mehrstimmigkeiten erzeugt werden, ähnliche Sachverhalte aus verschiedenen Perspektiven beleuchtet und Positionen (auch gegeneinander) in Stellung gebracht werden. Dramaturgisch lässt sich hier über die Bildbearbeitung (z. B. Schwarz-Weiß-Darstellung; Körnung des Bildmaterials) oder durch Akustik (z. B. Hall, Hintergrundgeräusche) zusätzlich in das Material eingreifen, um Atmosphären zu erzeugen, Betonungen vorzunehmen oder Kontrapunkte zu setzen.

Diesen Nutzungen von Originaldaten steht eine visuelle oder materiale Übersetzung der Ergebnisse durch Installationen/Ausstellungsarrangements diametral gegenüber. Hierbei handelt es sich um eine (künstlerische) Bearbeitung, die sowohl den Produzierenden ein Maximum an Interpretationsspielraum gibt, als auch den Rezipierenden die weitestgehende Deutungs-, Verstehens- und Aneignungsleistung abverlangt.

\subsection{Interviews im Gesamtarrangement der Ausstellung: Zum Stellenwert von Interviewmaterial}

Ungeachtet dessen, auf welche Art und Weise auf das Interviewmaterial zugegriffen wird und es Eingang in die öffentliche Präsentation findet, bietet es verschiedene Rezeptionsschichten. Selbst das Rohmaterial spricht nicht für sich und ist mitnichten eindeutig, denn sonst würden Interpretationen verbaler Daten nicht so aufwendig und vielschichtig ausfallen. Auch aufbereitete Daten, sei es in Form von Fallgeschichten oder Zitatenkollektionen, sind unterschiedlich eindeutig. Dennoch gilt, dass je nach Aufbereitung der Aussagegehalt in seiner Eindeutigkeit variiert und verschiedene Nachfragen (oder Irritationen) suggeriert.

Das Arrangement von Interviews in einem Ausstellungskontext ist zudem äußerst variabel: Porträtierungen als gestaltete »Poster « können die Hauptausstellung - auf 
Stellwänden oder gerahmt gehängt - bilden, oder wie im Kontext der DDR-Jugendkultur-Ausstellung als »blätterbares Buch « (im Poster-Blättergestell) arrangiert werden. Aufbereitete Audio-/Videoauszüge können über Kopfhörer angeboten werden und damit einen intimen Moment darstellen, in dem Privates anvertraut und die Möglichkeit geboten wird, in die Geschichte(n) »einzutauchen «. Oder die Botschaften werden über Lautsprecher hörbar öffentlich gemacht und $\gg$ in den Raum gestellt«, zum Teil sogar in einer Weise, dass ein polyphoner Charakter erzeugt wird, in dem die Aussagen akustisch interferieren. Von Einzelpersonen berücksichtigte Zitate oder markante Aussagen/Worte - mit oder ohne Nennung der Namen der Interviewten - können en passant dargeboten werden, eine Art inhaltliche Linie (Chronologie) vorgeben oder zu Themenclustern gruppiert werden.

$\mathrm{Zu}$ beachteten ist dabei aber, dass die Interviewpräsentationen in den drei von mir umgesetzten Ausstellungen in ein Gesamtarrangement aus verschiedenen Elementen eingebettet wurden. Sie stehen im Dialog mit der jeweiligen Raumgestaltung, den darin eingehenden Objekten und Dokumenten. Daraus lassen sich Konsonanzen wie auch Dissonanzen erzeugen. Die Ergebnisse der Ausstellung sind insofern am Ende mehr als die präsentierten Aussagen. Das Gesamtergebnis spricht die Bereiche des Sehens, Hörens, des Taktilen, des Olfaktorischen und Haptischen und damit alle Sinne an. Und sie eröffnen allen, in je individueller Weise » mitzumachen « und sich »berühren « zu lassen. Solche Einladungen sind zuweilen implizit, können aber auch sehr explizit gemacht werden und auf Einbezug und Partizipation zielen.

Von hier ausgehend eröffnet sich auch die Möglichkeit, die Ausstellungen selbst für weitere Daten- und Materialsammlungen zu nutzen. Aufgezeichnete Beobachtungen und Gespräche mit Besuchenden können zur Validierung und für Ergänzungen verwendet werden. Für die Ausstellung »Jugendkultur in Stendal: 1950-1990 « wurde dazu aufgerufen, weitere Dokumente im Museum abzugeben, um diese in die laufende Ausstellung zu integrieren (was aber letztlich aufgrund fehlender Kapazitäten nur ansatzweise realisiert wurde), die Idee einer $\gg$ Memorybox « - mit der Option, Videobotschaften und inhaltliche Beiträge selbst aufzuzeichnen - war technisch nicht umsetzbar; es wurde nur »konventionell « ein Gästebuch ausgelegt. Die flankierenden »Erzählcafés « wurden protokolliert und im Forschungskontext diskutiert, aber nicht weiter aufbereitet und dokumentiert.

\section{$5 \quad$ Geltungsbegründung}

Bei Ausstellungen als dem »Resultat « und dem aus einer spezifischen Übersetzungsarbeit hervorgehenden Produkt eines vorausgehenden Forschungsprojekts ist zu fragen, wie dieses bewertet werden kann. Innerhalb der qualitativen Forschung findet sich 
eine eingehende Debatte zur Geltungsbegründung, die sowohl die Gestaltung des Forschungsprojektes betrifft, aber auch die Güte von dessen Darstellung in Artikeln (Ilg und Boothe 2010). Wie aber verhält es sich mit der Einschätzung der Qualität von Ausstellungen?

$\mathrm{Zu}$ fragen ist zunächst, inwieweit Aussagen über die Anlage und die Umsetzung der Studie Eingang finden im Rahmen der Präsentation und wie mit den in der qualitativen Forschung verbundenen Fragen umgegangen wird; diese betreffen die Berücksichtigung von Fällen (Homogenität/Heterogenität des Samples), die Gestaltung der Interviewsituationen (Offenheit), die Aufbereitung (Transkription und Aufnahmebearbeitung) sowie die Auswertung (Bildung von Kategorien, Theorienentwicklung), zusätzlich sind noch die Einhaltung forschungsethischer Aspekte, die Frage der Rückkopplung (member check) und der Einbezug der Beforschten (als Mitforschende im Sinne partizipativer Ansätze) und die Frage der Involviertheit und damit verbunden die Relevanz von Selbstreflexivität/Subjektivität berührt (im Überblick: Flick 2017).

Bei den von mir realisierten wie auch bei allen erwähnten Ausstellungen ist nur zum Teil ein Einblick in die Forschungsarbeit selbst gewährt. Diese wird zumeist nur kurz in den Ausstellungsbeschreibungen (oder dem Begleitbuch/Ausstellungskatalog) genannt oder in der Ausstellung selbst auf dem sogenannten Super-A-Text skizziert; lediglich bei der »Angst(frei) «-Ausstellung wurde sehr offensiv die Bezogenheit - durch Einspielen von Auswertungssitzungen und Reflexionsmemos in einer Endlosschleife thematisiert.

Auch ist deutlich geworden, dass aufgrund der verschiedenen Formen der Darstellung Einblicke in die Datenkonstruktion (dies insbesondere bei der Verwendung von Rohmaterial) gewährt werden. Die aufbereiteten Materialpräsentationen liefern dagegen nur selten Hinweise auf die Rekonstruktionsarbeit; die Ergebnisse werden - ohne ein Making-of - anschaulich präsentiert. Denn bezogen etwa auf die vielfach genutzten Porträtierungen hat sich mittlerweile eine Text-Bild-Sprache etabliert, die ein durchaus »einfacheres « und mit Blick auf Kosten auch kalkulierbareres Output darstellt. Bei solchen Ergebnisaufbereitungen wird die - zum Teil aufwendige - Analysearbeit nicht dargestellt; sie sind überwiegend deskriptiv und geben anschaulich Einblicke in die untersuchten Lebenswelten.

Inwieweit hier ausgearbeitete Kategorien oder gar theoretische Zusammenhänge explizit gemacht werden, variiert erheblich. Je nach Zusammenschau - und durch die Art der Zusammenstellung - verschiedener Porträts lassen sich zumindest Eindrücke an Vielfalt, Typik, an Extremfällen andeuten, kurzum Gemeinsamkeiten und Unterschiede oder Entwicklungslinien markieren. Diese können mehr oder weniger explizit gemacht werden, je nachdem, ob etwaige ausgewiesene Überschriften eher thematische Bereiche anzeigen oder im Sinne herausgearbeiteter Kategorien formuliert angeboten 
werden; so wurde beispielsweise in der Ausstellung »Skulpturale Collagen « bei der Benennung der einzelnen Stationen und Installationen auf die Kategorienbezeichnungen zurückgegriffen.

$\mathrm{Ob}$ die angebotene Kollektion verschiedene Lesarten und damit Interpretationsangebote offeriert, hängt auch mit der Ausstellungsarchitektur zusammen und damit, welchem Narrativ die Ausstellung als Gesamtes und die ihr zugehörigen Teilbereiche folgen. Hierbei kann durchaus mit Erwartungshaltungen gespielt und die antizipierte Rezeption »infrage gestellt « - das meint: irritiert - werden.

Im Kontext der performativen Sozialwissenschaft werden dann auch andere Kriterien der Güte favorisiert; dies sind insbesondere Fragen der Resonanz. Das meint auf der einen Seite durchaus ein leicht messbares quantitatives Kriterium nach Anzahl erreichter Menschen. Zum anderen ein deutlich schwieriges Maß, nämlich die Beantwortung der Frage, ob die Präsentation die Menschen erreicht - und das meint »berührt « hat, es zu einer (eingehenden) Auseinandersetzung und sogar zu einem Perspektivwechsel gekommen ist (Schreier 2017).

Bei der Ausstellung »Jugendkultur in Stendal: 1950-1990 « waren es fast 2.500 Besuchende; dies ist für ein regionales Landesmuseum eine enorme Anzahl. Damit war sie seit Bestehen die nachgefragteste Ausstellung. Alleine bei der Vernissage waren über 200 Menschen anwesend, darunter sehr viele der Interviewten und Leihgebenden. Der enorme quantitative Zuspruch drückte sich auch qualitativ aus. Die Rückmeldungen während der Ausstellung, die - allerdings nicht systematischen - Beobachtungen während der Besuchszeiten wie auch die Einträge in das Gästebuch zeigten, dass es gelungen ist, eine »Geschichte « zu erzählen, die Erinnerungen mobilisierte, biografische Abgleiche eröffnete und in diesem Falle als eine Form der »Wertschätzung « für das eigene Leben erfahren wurde.

$\mathrm{Zu}$ fragen bleibt aber, wer mit einer Ausstellung erreicht wird, und inwieweit die Bezugnahme auf künstlerisch-ästhetische Mittel im doppelten Wortsinne »exklusiv « ist - und damit den Anspruch und Wunsch, möglichst viele Interessierte jenseits des » eigenen Universums « zu erreichen, konterkariert. ${ }^{8}$ Je experimenteller die Umsetzung, je subversiver die Darstellungsformen, desto eher wird performative Sozialforschung zelebriert, die sich einer einfachen (und damit eindeutigen) Aneignung widersetzt, aber eben auch weniger anschlussfähig und »leicht « verständlich (konsumierbar) ist. ${ }^{9}$

Ebenso bleibt zu fragen, inwieweit bereits beim Studiendesign aufgrund der Intention, die Ergebnisse öffentlich zu machen, Selektionen mit Blick auf das Sampling und auch hinsichtlich des Interviewberichts erfolgen. Das heißt, das Wissen darum, später »öffentlich « und »erkennbar « zu sein, kann das, was (nicht) gesagt wird (und wer sich überhaupt bereit erklärt, Teil des Samples zu sein), erheblich beeinflussen. Und auch die Interviewführenden können angesichts des Wissens, für eine spätere Präsentation Tex- 
te/Aussagen produzieren zu » müssen «, bereits die Erhebung steuern, um »prägnante « Sätze zu evozieren. Schließlich gilt zu beachten, dass zum Teil mit Klarnamen und Gesicht versehene Aussagen nicht immer öffentlich verwendet werden können aufgrund der behandelten Inhalte. Kurzum: Die je gegebenen Produktionsbedingungen und damit die darin sich zeitigende Konstruktion der erhobenen Daten gilt es zu reflektieren. Inwieweit darüber Auskunft gegeben werden kann, bleibt offen. Und zu bedenken gilt es auch, dass möglicherweise einer sehr überzeugenden Ausstellung nicht anzusehen ist, ob sie aus einem weniger herausragenden Forschungsprojekt hervorgeht - wie umgekehrt eine sehr gelungene Studie wenig ansprechend (uninspiriert) als Ausstellung umgesetzt werden kann. Über die Verwobenheit von Prozess und Produkt wird zukünftig mit Blick auf die Geltungsbegründung und den Güteanspruch im Zusammenhang mit performativer Sozialwissenschaft zu arbeiten sein. Hierzu wäre es notwendig, dass die an solchen Arbeiten Beteiligten - also Wissenschaftler*innen aus den Sozial- und Humanwissenschaften einerseits und Kulturschaffende und Vertreter*innen aus der Kunst andererseits - sich austauschen, denn performative Sozialwissenschaft meint nicht

»simply writing a poem or putting on a play merely because that happens to be a pastime (or frustration) of an academic. Rather, it is finding the right arts-based method to help answer the research question and/or to disseminate the findings to the public. Ideally, it is about forming collaborations with artists themselves and creating a professional learning and/or dissemination experience, which includes the wider community to engender a meaningful investment in the project, its outputs and outcomes « (Jones 2014, o. S.).

\section{Ausblick}

Auch wenn Ausstellungen schon seit jeher traditionell zur Wissensvermittlung gedient haben und sich seit Längerem Wissenschaftsausstellungen im Sinne eines »konstruktivistischen Museumsverständnisses « (Lepenies 2003) etablieren konnten, mehren sich Ausstellungen als Format, um wissenschaftliche Erkenntnisse für eine interessierte Öffentlichkeit aufzubereiten. Die Gründe dafür sind nicht zuletzt aufgrund der zunehmenden Bedeutung des »visuell Dargebotenen « allgemein zu sehen und liegen auch darin, dass die Kosten für die Erstellung von Postern und anderen »einfachen « Exponaten deutlich gesunken sind. Hinzu kommt, dass an Hochschulen zunehmend als Auftrag gesehen wird, neben den beiden traditionellen Säulen - Lehre und Forschung nunmehr auch verstärkt über die Möglichkeiten und Notwendigkeiten nachzudenken, Forschungsergebnisse in gesellschaftlichen Nutzen zu übersetzen - mithin sich als dritte Säule »Third Mission « zu etablieren scheint (Henke, Pasternack und Schmid 2017). 
In diesem Sinne sind auch die Bestrebungen etwa des BMBF zu lesen, die in Förderlinien zunehmend aufnehmen, im Anschluss - oder zum Teil auch schon parallel zum eigentlichen Forschungsprojekt - die Ergebnisse entsprechend für eine allgemeine Öffentlichkeit aufzubereiten.

Qualitative Forschung hat per se paradigmatisch dafür gestanden, nicht nur »alltagsnah « zu sein, sondern auch Konzepte bereitzuhalten, die es ermöglichen, die Beforschten einzubeziehen und Ergebnisse zurückzumelden. Über individuelle Formen bieten sich hier auch gerade öffentlichkeitswirksame Präsentationen an, bei denen die Beforschten als mitforschende Akteur*innen eingebunden werden.

Vor dem Hintergrund dieser Entwicklungen und den Anliegen und Ansprüchen qualitativer Forschung lohnt es sich, mehr über Ausstellungen als Präsentationsmodi in all ihren Vielgestaltigkeiten nachzudenken; dazu gehört auch, sich vermehrt um Fragen der Rezeption und Wirkung zu kümmern; aber noch mehr, daran zu arbeiten, wie ein Making-of transparenter gemacht werden kann, um die Gestaltung und Umsetzung des Forschungsprozesses in eine Ausstellungsrealisation einbinden zu können, auch wenn klar ist, dass eine für Studien herangezogene Bemessung der Güte nur teilweise einlösbar scheint. Denn eine Präsentation von Ergebnissen aus einem Forschungsprozess ist nicht der Prozess. So gilt insgesamt, dass im Rahmen performativer Sozialwissenschaft, die für die gelungene Verknüpfung von Kunst und Wissenschaft stehen soll, eine Wissenschaftsausstellung so wenig eine Kunstausstellung ist, wie andere Realisierungen im Zuge von Arts-informed und Arts-based Research weder (nur) Kunst noch (nur) Wissenschaft sind. Handelt es sich doch stets um Hybride.

\section{Anmerkungen}

1 Einige der Darlegungen gehen zurück auf meine Ausführungen zur performativen Sozialwissenschaft in der Psychologie (Mey 2018a, dort insbesondere die Abschnitte 2 und 3.4).

2 Festival-Kurator: Ludger Lemper; Intendant: Dirk Löschner, Dramaturg: Sascha Löschner.

3 Oberspielleiter: David Lenard.

4 Für eine Präsentation im Rahmen der "Langen Nacht der Wissenschaften« in Magdeburg wurden die auf Stahlgerüste aufgebauten Monitore zu einem Dreieck arrangiert, sodass in der Mitte stehend alle Stimmen - der in acht Stunden in Endlosschlaufe präsentierten Videointerviewcollagen - zu hören waren, je näher sich die Zuschauenden auf einen Monitor zubewegten, dann die Aussagen des jeweiligen Themenbereichs separat vernommen werden konnten.

5 Die Erzählcafés waren als intergenerationale Dialogforen angelegt, bei denen pro vorgestelltes Jahrzehnt zwei der Interviewten nochmals ihre Erfahrungen Revue passieren ließen, um dann das Auditorium einzubeziehen, das Fragen stellen oder seinerseits Erlebnisse einbringen konnte. Die Erzählcafés wurden in Absprache mit der lokalen Presse durchgeführt und durch Vor- und Nachberichte begleitet. https://ausstellung-jugendkultur-stendal-1950-1990.h2.de (zugegriffen am 18.02.2020). 
7 Eine ähnlich ausgerichtete Ausstellungsarchitektur wird aktuell aus einem laufenden Projekt unter dem Titel »Kunst in der Altmark. Anders Sehen« für Präsentationen im kommenden Jahr im Kunsthaus Salzwedel und in der Landesvertretung Sachsen-Anhalt in Berlin konzipiert, in deren Rahmen fast 20 bildende Künstler*innen interviewt wurden. https://www.h2.de/ hochschule/fachbereiche/angewandte-humanwissenschaften/forschung/kunst-in-der -altmark.html (zugegriffen am 18.02.2020).

8 Dieser Gedanke geht auf eine Frage von Ken Horvath im Anschluss meines an der Universität Luzern gehaltenen Vortrags (03.11.2018) zurück. Irene Leser $(2019$, S. 211) weist in diesem Zusammenhang auf Befunde des Statistischen Bundesamtes hin, wonach nur knapp zwei Prozent der deutschen Bevölkerung regelmäßig Museen, Ausstellungen und Galerien besuchen.

9 Eine Distinktion, die mir wichtig ist und welche ich erstmals im Rahmen einer "performativen Aussprache« im Nachgang meines Vortrages am Zentrum für Performance Studies der Universität Bremen (11.01.2018) expliziert habe, nachdem mich Simon Makhali (Theater der Versammlung zwischen Bildung, Wissenschaft und Kunst) nach den Grenzen performativer Sozialwissenschaft fragte.

\section{Literatur}

Barone, Tom und Elliot W. Eisner. 2012. Arts-based research. Thousand Oaks, CA: Sage.

Böhm, Birgit, Heiner Legewie und Hans-Liudger Dienel. 2008. »Die Bürgerausstellung: Eine Kombination sozialwissenschaftlicher, partizipativer und künstlerischer Elemente«. Forum Qualitative Sozialforschung/Forum: Qualitative Social Research 9 (2): Art. 33. http://dx.doi.org/10. 17169/fqs-9.2.380.

Breuer, Franz, Arnuf Deppermann, Udo Kuckartz, Günter Mey, Katja Mruck und Jo Reichertz. 2014. »All is data - Qualitative Forschung und ihre Daten. Eine Diskussion«. In Qualitative Forschung: Analysen und Diskussionen, hrsg. v. Günter Mey und Katja Mruck, 261-90. Wiesbaden: Springer VS.

Chamberlain, Kerry, Kathryn McGuigan, David Anstiss und Kayla Marshall. 2018. »A change of view: arts-based research and psychology«. Qualitative Research in Psychology 15 (2-3): 131-39. http://dx.doi.org/10.1080/14780887.2018.1456590.

Church, Kathryn. 2008. „Exhibiting as inquiry. Travels of an accidental curator«. In Handbook of the arts in qualitative research, hrsg. v. Gary Knowles und Ardra L. Cole, 421-34. Thousand Oaks, CA: Sage.

Dresing, Thorsten und Thorsten Pehl. 2018. Praxisbuch Interview, Transkription \& Analyse. Anleitungen und Regelsysteme für qualitativ Forschende. 8. Aufl. Marburg: auiotranscription. Zugegriffen am 10.10.2019. https://www.audiotranskription.de/downloads\#Praxisbuch.

Faulkner, Sandra L. 2009. Poetry as method: Reporting research through verse. Walnut Creek, CA: AltaMira.

Field, Hyman und Patricia Powell. 2001. »Public understanding of science versus public understanding of research «. Public Understanding of Science 10 (4): 421-26.

Flick, Uwe. 2017. »Gütekriterien qualitativer Forschung in der Psychologie«. In Handbuch Qualitative Forschung in der Psychologie, 2. erw. und akt. Aufl., hrsg. v. Günter Mey und Katja Mruck. Heidelberg: Springer Reference Psychologie. https://doi.org/10.1007/978-3-658-18387-5_ 30-1.

Funch, Bjarne Sode (2015). »The creation of an art exhibition«. [Roskilde.] Unveröffentlichtes Manuskript. 
Gergen, Mary und Ken J. Gergen. 2010. »Performative social science and psychology«. Forum Qualitative Sozialforschung/Forum: Qualitative Social Research 12 (1): Art. 11. http://dx.doi.org/10. 17169/fqs-12.1.1595.

Gläser, Jochen und Grid Laudel (2010). Experteninterviews und qualitative Inhaltsanalyse: als Instrumente rekonstruierender Untersuchungen. 4. Aufl. Wiesbaden: VS.

Helfferich, Cornelia. 2011. Die Qualität qualitativer Daten. Manual für die Durchführung qualitativer Interviews. 4. Aufl. Wiesbaden: VS.

Henke, Justus, Peer Pasternack und Sarah Schmid. 2017. Mission, Die dritte: Die Vielfalt jenseits hochschulischer Forschung und Lehre: Konzept und Kommunikation der Third Mission. Berlin: Berliner Wissenschafts-Verlag.

Ilg, Stefan und Brigitte Boothe. 2010. »Qualitative Forschung im psychologischen Feld: Was ist eine gute Publikation?«. Forum Qualitative Sozialforschung/Forum: Qualitative Social Research 11 (2): Art. 25. http://dx.doi.org/10.17169/fqs-11.2.1371.

Johnson, Helen, Emily Carson-Apstein, Simon Banderob und Xander Macualay-Rettino. 2017. »You kind of have to listen to mes: Researching discrimination through poetry«. Forum Qualitative Social Research/Forum Qualitative Sozialforschung 18 (2): Art. 6. http://dx.doi.org/10.17169/ fqs-18.3.2864.

Jones, Kip. 2014. »What is performative social science? The potential of arts-based research and dissemination«. Zugegriffen am 10.10.2019. http://discoversociety.org/2014/05/06/what-is -performative-social-science-the-potential-of-arts-based-research-and-dissemination.

Jones, Kip. 2017. »Performative Social Science«. In International encyclopedia of communication research methods, hrsg. v. Jörg Matthes. Hoboken, NJ: John Wiley \& Sons, Inc. Zugegriffen am 10.10.2019. http://eprints.bournemouth.ac.uk/22616/.

Jones, Kip, Mary Gergen, John J. Guiney Yallop, Irene Lopez de Vallejo, Brian Roberts und Peter Wright, Hrsg. 2008. Performative social science/Performative Sozialwissenschaft. Forum Qualitative Sozialforschung/Forum: Qualitative Social Research 9 (2). Zugegriffen am 10.10.2019. http://www.qualitative-research.net/index.php/fqs/issue/view/10.

Keppler, Dorothee, Birgit Böhm und Hans-Liudger Dienel, Hrsg. 2013. Die Bürgerausstellung. Die Perspektive von Bürgern und Bürgerinnen als Gegenstand qualitativer Sozialforschung und praktischer Beteiligung. München: oekom.

Knowles, Gary und Ardra L. Cole, Hrsg. 2008. Handbook of the arts in qualitative research. Thousand Oaks, CA: Sage.

Kostas, Gabriele und Irene Ziehe. 2016. Europas neue Alte. Ein foto-ethnografisches Projekt. Berlin. Nicolai. Leavy, Patricia, Hrsg. 2017. Handbook of arts-based research. New York: Guilford Press.

Legewie, Heiner. 1994. »Globalauswertung von Dokumenten«. In Texte verstehen: Konzepte, Methoden, Werkzeuge, hrsg. v. Andreas Boehm, Andreas Mengel und Thomas Muhr, 177-82. Konstanz: UVK. Zugegriffen am 10.10.2019. https://nbn-resolving.org/urn:nbn:de:0168-ssoar-14547.

Legewie, Heiner. 2003. Erzählungen und Bilder der Stadt: Lebensqualität und Tourismus in historischen Vierteln von Florenz und Berlin. Berlin: TUB/ZTG.

Legewie, Heiner und Georg Eichinger. 2017. Künstler in Athen - Stadt der Krise. Berlin: Verlag der TU Berlin.

Lepenies, Annette. 2003. Wissen vermitteln im Museum. Köln: Böhlau.

Leser, Irene. 2019. "Science meets art. Zu den Spielarten performativer Sozialforschung«. In Bildung und Erziehung in der ästhetischen Gegenwart, hrsg. v. Clemens Bach, 191-221. Wiesbaden: Springer VS. http://dx.doi.org/10.1007/978-3-658-21891-1_9.

Leser, Irene und Günter Mey. 2017. »Man muss der Jugend etwas beaten! Jugendkultur in der ländlichen Region«. Diskurs Kindheits- und Jugendforschung 12 (1): 95-101. https://doi.org/ 10.3224/diskurs.v12i1.08. 
Mey, Günter. 2000. »Erzählungen in qualitativen Interviews: Konzepte, Probleme, soziale Konstruktionen«. Sozialer Sinn. Zeitschrift für hermeneutische Sozialforschung 1: 135-51. Zugegriffen am 10.10.2019. http://nbn-resolving.de/urn:nbn:de:0168-ssoar-4471.

Mey, Günter. 2011. »Angst - Intimes Bekenntnis im öffentlichen Raum. Chancen einer performativen Sozialwissenschaft«. Theater der Zeit (Beilage) 12: 29-32.

Mey, Günter. 2018a. »Performative Sozialwissenschaft und psychologische Forschung«. In Handbuch Qualitative Forschung in der Psychologie, 2., akt. u. erw. Aufl., hrsg. v. Günter Mey und Katja Mruck. Heidelberg: Springer Reference Psychologie. https://doi.org/10.1007/978-3-658-18387-5_29-1.

Mey, Günter, Hrsg. 2018b. Jugendkultur in Stendal: 1950-1990. Berlin: Hirnkost.

Mey, Günter und Katja Mruck. 2009. »Methodologie und Methodik der Grounded Theory«. In Forschungsmethoden der Psychologie. Zwischen naturwissenschaftlichem Experiment und sozialwissenschaftlicher Hermeneutik, Band 3, hrsg. v. Wilhelm Kempf und Marcus Kiefer, 100-52. Berlin: Regener.

Mruck, Katja, Stefan Gradmann und Günter Mey. 2004. „Open Access: Wissenschaft als Öffentliches Gut«. Forum Qualitative Sozialforschung/Forum: Qualitative Social Research 5 (2): Art. 14. http://dx.doi.org/10.17169/fqs-5.2.624.

Mruck, Katja und Günter Mey. 1998. „Selbstreflexivitat und Subjektivitat im Auswertungsprozess biographischer Materialien - zum Konzept einer `Projektwerkstatt qualitativen Arbeitens « zwischen Colloquium, Supervision und Interpretationsgemeinschaft«. In Biographische Methoden in den Humanwissenschaften, hrsg. v. Gerd Jüttemann und Hans Thomae, 284-306. Weinheim: Beltz. Zugegriffen am 10.10.2019. https://nbn-resolving.org/urn:nbn:de:0168-ssoar-1200.

Prendergast, Monica, Carl Leggo und Pauline Sameshima, Hrsg. 2009. Poetic inquiry. Rotterdam: Sense Publishers.

Richard, Birgit und Heinz-Hermann Krüger, Hrsg. 2012. Megacool 4.0: Jugend und Kunst. Bielefeld: Kerber.

Ricœur, Paul. 1981. »Mimesis and representation. Annals of Scholarship«. Metastudies of the Humanities and Social Sciences 2: 15-32.

Schreier, Margrit. 2017. »Kontexte qualitativer Sozialforschung: Arts-Based Research, Mixed Methods und Emergent Methods«. Forum Qualitative Sozialforschung/Forum: Qualitative Social Research 18 (2): Art. 6. http://dx.doi.org/10.17169/fqs-18.2.2815.

Straub, Jürgen. 1999. Handlung, Interpretation, Kritik. Grundzüge einer textwissenschaftlichen Handlungs- und Kulturpsychologie. Berlin: de Gruyter.

Sullivan, Graeme. 2010. Art practice as research. Inquiry in visual arts. 2. Aufl. Thousand Oaks, CA: Sage.

Theater der Altmark. 2011. Angst(frei) Festival. Theater der Altmark, Stendal 03.09.-11.09.2011. Theater der Zeit (Beilage) 12.

Von Unger, Hella. 2017. »Forschungsbeziehungen in qualitativer Forschung: Gestaltung, Analyse und forschungsethische Reflexion«. Mittagsvorlesung im Rahmen des Berliner Methodentreffens Qualitative Forschung, 21.07.2017. Zugegriffen am 10.10.2019. http://www.berliner -methodentreffen.de/archiv/video/mittagsvorlesung_2017/.

Winter, Rainer. 2010. »Ein Plädoyer für kritische Perspektiven in der qualitativen Forschung«. Forum Qualitative Sozialforschung/Forum: Qualitative Social Research 12 (1): Art. 7. http://dx.doi.org/ 10.17169/fqs-12.1.1583.

Witzel, Andreas. 2000. „Das problemzentrierte Interview«. Forum Qualitative Sozialforschung/Forum: Qualitative Social Research 1 (1): Art. 22. http://dx.doi.org/10.17169/fqs-1.1.1132.

Witzel, Andreas und Herwig Reiter. 2012. The problem-centred interview. London: Sage.

Ziehe, Irene und Edgar Zippel. 2013. I'm not afraid of anything. Porträts junger Europäer. Stuttgart: Kehrer. 


\section{Der Autor}

Günter Mey, Prof. Dr. habil., ist Professor für Entwicklungspsychologie an der Hochschule Magdeburg-Stendal, Leiter des Instituts für Qualitative Forschung in der Internationalen Akademie Berlin sowie Privatdozent an der Kulturwissenschaftlichen Fakultät der Universität Bayreuth. Seine Arbeitsschwerpunkte sind qualitative Methodologie und performative Sozialwissenschaft sowie Biografie, Identität, Kultur, Transgenerationalität in den Themenfeldern Kindheit, Jugend und Alter. Weitere Informationen unter: http://www.humanwissenschaften.h2.de/l/ mey

Kontakt: Prof. Dr. habil. Günter Mey, Hochschule Magdeburg-Stendal, Angewandte Humanwissenschaften, Osterburger Str. 25, D-39576 Hansestadt Stendal, E-Mail: guenter.mey@h2.de 